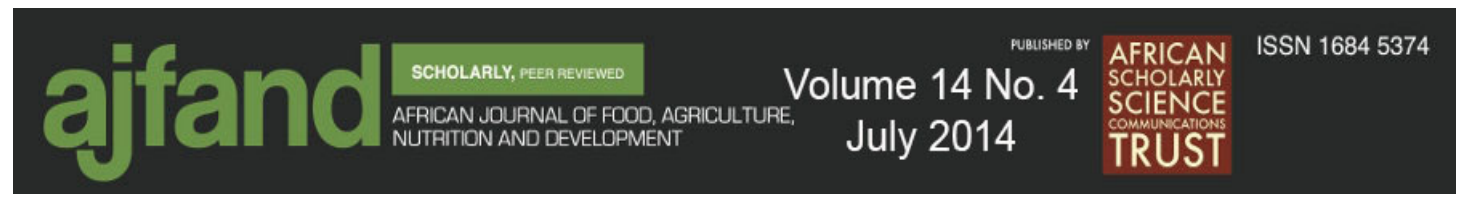

\title{
SIX MONTHS OF EXCLUSIVE BREASTFEEDING RECOMMENDATION: HOW APPLICABLE IS THE UNIVERSAL EXCLUSIVE BREASTFEEDING RECOMMENDATION POLICY?
}

\section{Ssemukasa EL ${ }^{* 1}$ and $\mathrm{J}$ Kearney ${ }^{2}$}

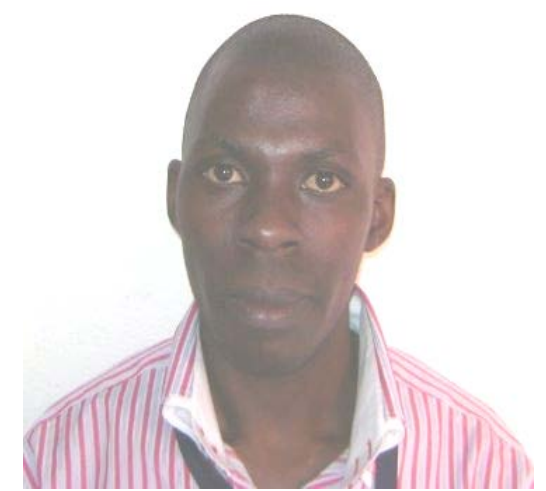

Edward Ssemukasa

*Corresponding author’s email: lsedward81@yahoo.com

${ }^{1}$ Present address: Lecturer in Engineering of Biological Materials, Department of Agro-Processing Engineering, Busitema University, P.O. Box 236, Tororo, Uganda

${ }^{2}$ John Kearney, Lecturer in Nutrition and Epidemiology, Department of Biological Sciences, Department of Biological Sciences, Dublin Institute of Technology, Dublin, Ireland. 


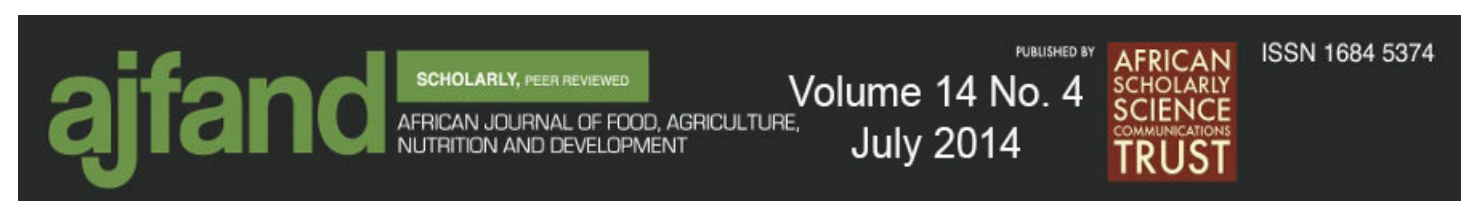

ABSTRACT

Public health organisations, including the World Health Organisation recommend 6 months of exclusive breastfeeding for optimal growth, cognitive development and health. In addition, the provision of nutritionally adequate and safe complementary foods to the infants while breastfeeding continues up until 2 years of age and beyond is also recommended. Exclusive breastfeeding for the first 6 months of life meets the energy and nutrient needs of the infants. The objective of this review therefore, is to assess the appropriateness of a universal 6 months exclusive breastfeeding recommendation policy in both developed and developing countries. In the last years, recommendations for the optimal duration of exclusive breastfeeding promoted by WHO and UNICEF started to differ. The World Health Organization had recommended exclusive breastfeeding for 4 to 6 months, with the introduction of complementary foods thereafter, whereas UNICEF preferred the wording "for about 6 months". This led to concerns in larger infant nutrition and public health communities. The American Academy of Pediatrics' position was also unclear: In two different sections of their Pediatric Nutrition Handbook, recommending human milk "as the exclusive nutrient source for babies during the first 6 months" and "the delayed introduction of solid foods until 4 to 6 months". Until recently, the only scientific evidence that contributed to the exclusive breastfeeding duration debate was based on observational studies, with well-recognized sources of potential bias. Reverse causality was another potential source of bias, particularly with respect to infectious morbidity and neuro-motor development. Infants who developed a clinically important infection were likely to become anorectic and experienced reduced breast milk intake that led to reduction in milk production and even termination of breastfeeding. The lack of functional and effective food safety standards and policies, safe drinking water, exclusive breastfeeding promoting programs, high infection rates and the broken healthcare systems in the developing countries unlike in the developed countries, are key areas that need further research before the 6 months recommended duration of exclusive breastfeeding can be reduced and/or changed to 4 months, to avoid child morbidity and mortality. It is also important that the 6 months of exclusive breastfeeding are promoted in all developing countries and 4-6 months in all developed countries.

Key words: Exclusive breastfeeding, universal recommendation policy 


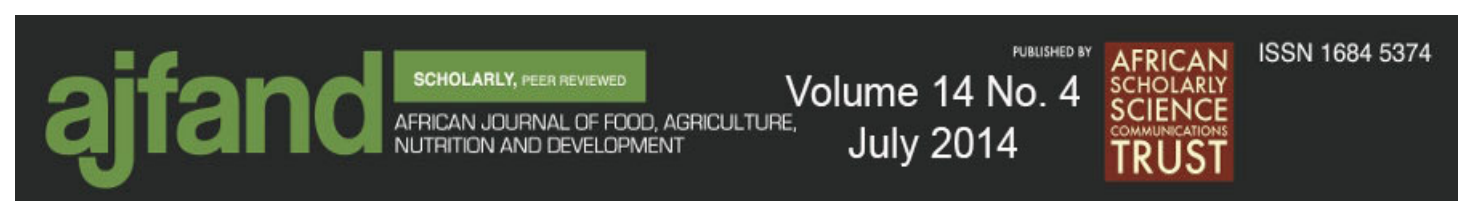

\section{INTRODUCTION}

Exclusive breastfeeding for 6 months is the optimal method of infant feeding [1]. Breastfeeding provides infants with nutrients for healthy growth, development and enhances immune systems. There are multiple lines of evidence to prove that exclusive breastfeeding for 6 months provides infants with protection against gastrointestinal infections and iron deficiency anemia [2]. It also keeps women amenorrhic (absence of two consecutive days of bleeding or spotting after postpartum day number 56 or 2 months) for 6 months postpartum and helps them to avoid unplanned pregnancies that end up in abortions. Moreover, research evidence shows that breastfeeding promotes sensory and cognitive development while protecting infant against chronic diseases, respiratory infections (flu, cough or pneumonia), and speeds up recovery from illnesses and reduces infant mortality [3, 4].

The WHO and UNICEF's global recommendations for optimal infant feeding recommends exclusive breastfeeding for 6 months and provision of nutritionally adequate and safe complementary foods starting from the age of 6 months with continued breastfeeding up to 2 years of age or beyond [5]. Worldwide, it is estimated that only 34.8\% of infants are exclusively breastfed for the first 6 months of life, with the majority receiving some other food or fluid in the early months [6]. Exclusive breastfeeding for the first 6 months of life meets the energy and nutrient needs of the infants [7]. Moreover, data collected from 64 countries covering $69 \%$ of births in the developing world suggest that there have been improvements in the rate of exclusive breastfeeding [8]. Between 1996 and 2006, the rate of exclusive breastfeeding for the first 6 months of life increased from 33\% to 37\%. Significant increases were made in sub-Saharan Africa, where rates increased from 22\% to 30\%, and Europe, where rates increased from $10 \%$ to $19 \%$. In Latin America and the Caribbean, excluding Brazil and Mexico, the percentage of infants exclusively breastfed increased from $30 \%$ in 1996 to $45 \%$ in 2006 [8].

Socio-demographic influences play a major role in explaining the low rates of exclusive breastfeeding. The importance of mothers' social groups and networks in affecting the infant feeding beliefs and practices should never be underestimated [9]. Depression arising from sickness affects infant feeding choices since depressed women may doubt their ability to feed the infants leading to early termination of breastfeeding efforts [10]. Moreover, early return to work is also a significant factor in the decision whether or not to breastfeed and thus a more conducive work environment (providing breastfeeding mothers with nurseries, breast milk expressing equipment and longer maternity leave incentives) with regard to breastfeeding might encourage the practice [11].

However, there is some evidence suggesting that formal support, provided by health professionals, may positively influence breastfeeding duration. This should be from competent professionals and the information should be consistent. Interventions such as group education sessions during antenatal checkups and individual counselling 


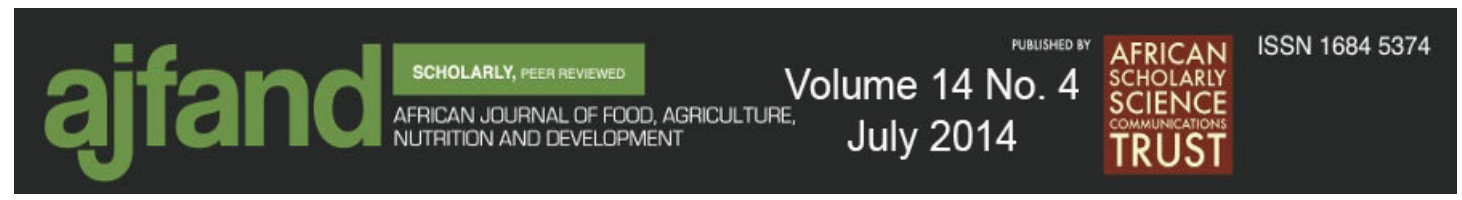

before and after birth may assist in improving exclusive breastfeeding duration [12, 13].

\section{Is 6 months of exclusive breastfeeding in developed countries appropriate?}

In the last years, recommendations for the optimal duration of exclusive breastfeeding promoted by WHO and UNICEF started to differ. The World Health Organization had recommended exclusive breastfeeding for 4 to 6 months, with the introduction of complementary foods thereafter, whereas UNICEF preferred the wording "for about 6 months" $[14,15]$. This led to concerns in larger infant nutrition and public health communities [16]. The American Academy of Pediatrics' position was also unclear: In two different sections of their Pediatric Nutrition Handbook, recommending human milk "as the exclusive nutrient source for babies during the first 6 months" and "the delayed introduction of solid foods until 4 to 6 months" [17]. Until recently, the only scientific evidence that contributed to the exclusive breastfeeding duration debate was based on observational studies, with well-recognized sources of potential bias. Some of these biases tended to favor exclusively breastfed infants, while others favored those who received earlier complementary feeding.

Infants who continued to be exclusively breastfed tended to be those who remained healthy and on an accepted growth trajectory $[18,19]$. Confounding by indication was another important bias, and operated in either direction [20]. Poorly-growing infants (especially those in developing countries) were likely to receive complementary foods earlier because of their slower growth. In developed countries, however, rapidlygrowing infants needed more energy than could be met by the increasingly spaced feedings typical of such settings. This resulted in crying and poor sleeping, supplementation with formula and/or solid foods, reduced suckling and a vicious cycle that led to earlier termination of breastfeeding.

Reverse causality was another potential source of bias, particularly with respect to infectious morbidity and neuro-motor development [21]. Infants who developed a clinically important infection were likely to become anorectic and experienced reduced breast milk intake that led to reduction in milk production and even termination of breastfeeding. This was particularly a problem in cross-sectional studies, because the temporal sequence of the early signs of infection and termination of breastfeeding could not be adequately appreciated; infection might have been blamed on the termination of breastfeeding, rather than the reverse. Advanced neuromotor development might also have lead to earlier induction of solid foods, which could have then received credit for accelerating motor development [22].

Moreover, other unmeasured or poorly measured confounding variables could also have biased the association between the introduction of complementary foods and infant health outcomes. Because of these well-recognized problems in observational studies, two controlled clinical trials from Honduras attracted considerable interest $[23,24]$. These trials allocated infants born to either continue breastfeeding exclusively for 6 months or to receive solid foods along with continued breastfeeding 


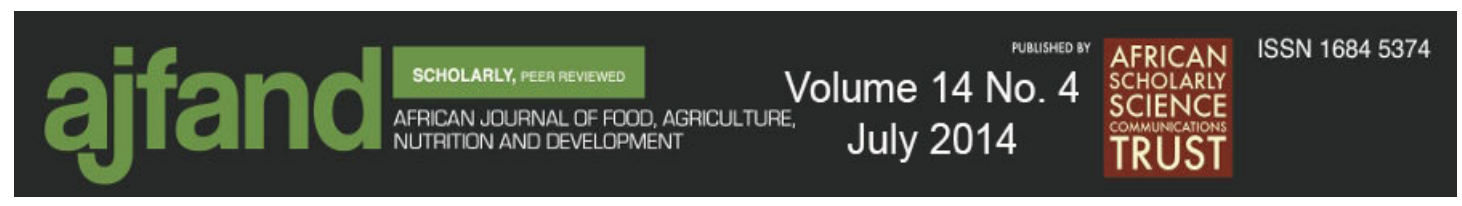

from 4 months onwards. However, the results showed neither significant benefit for growth nor any disadvantage for morbidity with the earlier introduction of complementary foods. The small sample sizes and published analyses based on compliance with allocation had prevented universal acceptance of these results [25].

Furthermore, the complementary foods used were those commonly found in developed countries, rather than those traditionally used in Honduras or other developing countries. Moreover, there had been an underlying assumption in this field that "one size fits all", which meant, that the average population effects could be applied to individual infants and that one international recommendation was therefore adequate for all infants. There had also been little discussion of the fact that all infants, regardless of how they had been fed, needed careful monitoring for growth and illness, with appropriate interventions undertaken whenever clinically indicated.

Because there was an ongoing controversy and polarization over this issue, the World Health Organization requested, in the spring of 2000, a systematic review of the available evidence before considering a revision or continuation of the then infant feeding recommendations. This resulted in three separate strata for considering the results of the studies located by the literature search: (1) controlled trials of exclusive versus mixed breastfeeding for 4-6 months from developing countries, (2) observational studies of exclusive versus mixed breastfeeding for 3-7 months from developing countries and (3) observational studies of exclusive versus mixed breastfeeding for 3-7 months from developed countries.

The study however, found no objective evidence of a "weanling's dilemma”. Besides the reduced morbidity due to gastrointestinal infection, the infants that had been breastfed exclusively for 6 or more months had no observable deficits in growth, and their mothers were more likely to remain amenorrhic. No benefits of introducing complementary foods between 4 and 6 months had been demonstrated, with the exception of improved iron status in one developing country setting (Honduras).

Moreover, the latter benefit could be achieved more effectively, through medicinal iron supplementation, (for example, administration of vitamin drops) it did not appear to justify the adverse effects of liquid or solid food supplementation on infectious morbidity, and lactational amenorrhea. Therefore, with that caveat, individual infants had to be managed individually, so that insufficient growth or other adverse outcomes were not ignored and appropriate interventions had to be provided. The available evidence demonstrated no apparent risks in recommending, as a general policy, exclusive breastfeeding for the first 6 months of life in both developing and developed country settings. However, large and rigorous cluster-randomized trials would help to resolve residual uncertainties about the possible advantages and disadvantages of the exclusive breastfeeding policy in both developed and developing countries.

Therefore, over the last 3 decades, the pediatric world agreed with the United Nations International Children Education Fund (UNICEF) and the World Health Organization (WHO) on the general recommendation to exclusively breastfeed healthy infants for 6 


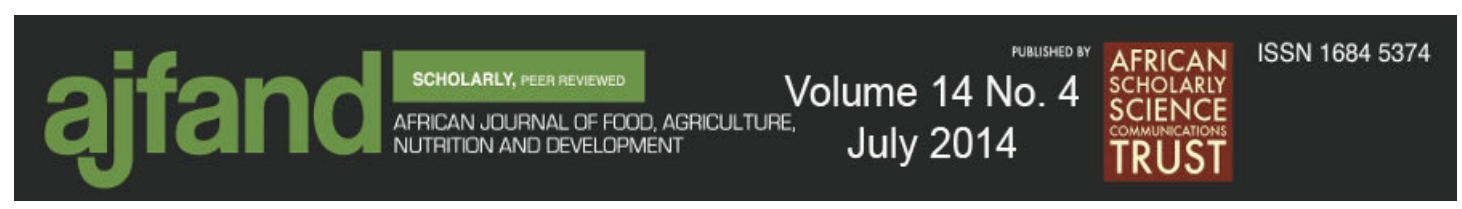

months and starting complementary feeding in the seventh month [26]. A consensus conference assembled by the late British pediatrician David Baum in 1988 in Bristol, United Kingdom, brought together the evidence for the health strengthening effect of exclusive breastfeeding for 6 months (unpublished data).

Recent research also found beneficial effects of lengthy exclusive breastfeeding [27]. The WHO produced the "Code of Marketing for Breast milk Substitutes" in 1980 and endorsed the point with the "Innocenti Declaration" in 2007. The WHO further included the recommendation as an enrollment criterion for its multi-continental study that led to the publication in 2006 of a global set of anthropometric references for child growth from birth to 6 years of age [28]. Besides the United Nations organizations, the non-governmental World Alliance of Breastfeeding Associations and the International Baby Food Action Group made the recommendation of 6 months exclusive breastfeeding as a basis for their advocacy and education activities as did governments who developed breastfeeding policies.

It is the consensus that 6 months of exclusive breastfeeding is having a protective effect against infectious diseases that this recommendation is based. However, studies from developed countries indicated that children should start receiving complementary foods (especially gluten-containing and potentially allergenic foods) when they still received breast milk. The epidemiological studies pointed to the observation that this combination of being breastfed and receiving complementary foods had a higher potential to prevent atopy and asthma. According to standard evidence criteria the findings were declared to meet evidence-level grade $C$. These findings from studies on breastfeeding and its impact on the incidence of atopic and allergic diseases led to a recommendation of early introduction of complementary foods in the fifth and sixth months that had been endorsed by the European Society of Pediatric Gastroenterology, Hepatology and Nutrition [29]. There were several challenges that arose with the change in exclusive breastfeeding policy recommendations in developed countries and the most challenging issues were:

1. If we needed to adjust breastfeeding recommendations to the socioeconomic situation of the families or to national priorities or give priority to the change in disease pattern observed in developed countries?

2. How best the change could be communicated from the-up until now-uniform breastfeeding message toward a differentiating message?

3. And how the children growing up in resource-poor settings and benefiting from 6 months exclusive breastfeeding could be protected from the early introduction of complementary foods?

Moreover, some studies suggested that the early introduction of complementary foods might prevent atopy. The studies which had been performed in settings in which most mothers did not exclusively breastfeed for 6 months and also had short periods of total exclusive breastfeeding and further in settings with breastfeeding over 6 months such 


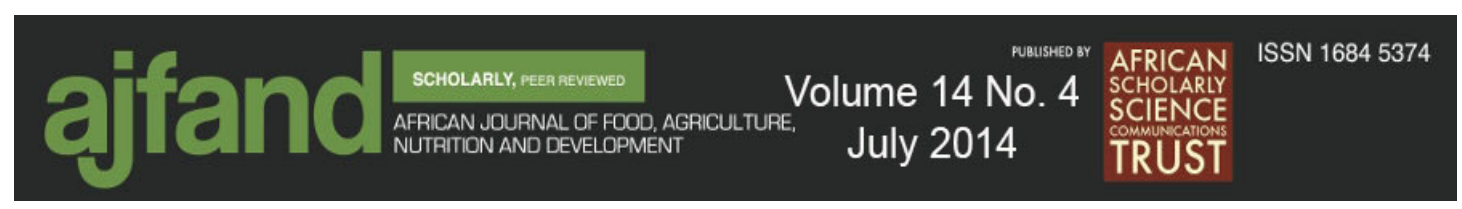

as Brazil, the protective effect of 6 months of exclusive breastfeeding against atopy had been demonstrated [30]. This therefore, served to suggest that it was not the early introduction of complementary foods exerting a protective effect but the introduction under continuing breastfeeding [31]. If this is true, exclusively breastfeeding for 6 months and introduction of complementary foods with continued breastfeeding should be promoted. Additionally, recent studies from developed countries showed lower infection rates and lower Asthma even after 3 months of exclusive breastfeeding [32, 33].

The dilemma of breastfeeding policies in the last 50 years had been that the rate of women starting to breastfeed their babies had been increasing enormously, but most of these women did not exclusively breastfeed for 6 months and many stopped breastfeeding entirely before the baby was 6 months old [34]. This challenge of effectively promoting exclusive and partial breastfeeding still exists and the question of "why more women do not follow the recommendation" cannot be answered only by pointing to the breast milk substitutes industry and its marketing strategies. Questions about the effectiveness of breastfeeding policies and the conditions in the family, in the community, and in the society that enable women to breastfeed or prevent them from nursing their children are seriously under-researched [35]. Addressing these questions requires widening the scope by not just looking at the benefits to the infant but the benefits of supporting the mother-child dyad.

Moreover, women's rights and status have been found to be of great importance for the prevention of childhood malnutrition. It is time to conduct research accordingly, on promoting the effects of breastfeeding that do not take into account the real duration of breastfeeding before setting up generalized new recommendations [36]. The priority should be on promoting 6 months exclusive breastfeeding and, although there is no evidence shown yet, those children in developed countries who are breastfed for a short time only should receive complementary foods before 6 months of age as long as they are still breastfed.

\section{Should the duration of 6 months of exclusive breastfeeding in developing and developed countries remain the same?}

Much as the WHO recommends exclusive breastfeeding for 6 months, the universal policy to exclusively breastfeed in developing and developed countries is another area that is under- researched.

The developed countries have fully functional and effective food safety standards and policies unlike the developing countries. Advocating for a unified exclusive breastfeeding recommendation for both developed and developing countries calls for unified and functional food safety standards and policies in developing countries where they are non-existent. However, this comes at an extra resource and financial investment. The resource-restricted communities especially those in developing countries would need donor funding to enforce a unified exclusive breastfeeding 


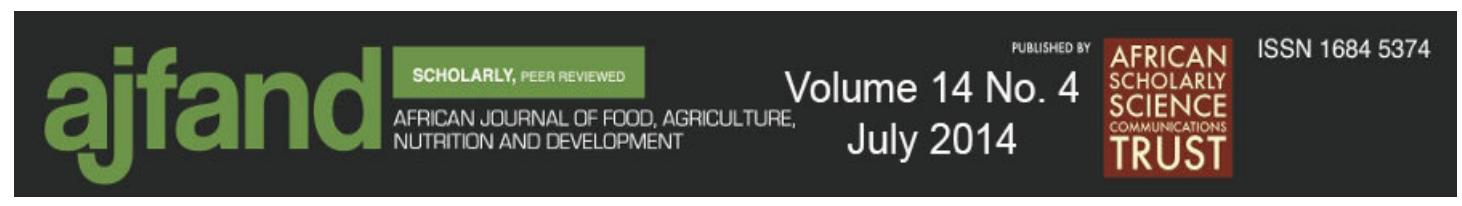

policy which process is bureaucratic and lengthy that it is unlikely that the poor communities will meet in the set deadlines.

Moreover, the developing countries are still struggling to effectively implement the 6 months recommendation of exclusive breastfeeding since its induction in 2001. The developed countries, unlike the developing countries, therefore, don't necessarily have to be locked to the 6 months of exclusive breastfeeding since their food safety standards and policies can ensure safe complementary foods for the infants and can invest enough resources into research just in case there was need to revisit the exclusive breastfeeding policy in force. Contrary to that, the limitations in research and resource investments in developing countries, the lack of mineral and micronutrient supplementation programs and the failures in meeting the deadlines in infant feeding research, may result in confusion among most breastfeeding mothers and lead to increased child mortality.

The developed countries have a fully developed, functional (stocked medicines and medical staff) healthcare system, proper sanitation and hygiene practices that are operational unlike in the developing countries where access to healthcare is low and the functionality and operation of the healthcare system is broken and almost nonexistent. Moreover, recent studies from developed countries indicate lower infection rates and lower asthma even after 3 months of exclusive breastfeeding [32, 33]. However, the rates of infections in developing countries are higher. Exclusive breastfeeding for 6 months protects against infections, which is critical in developing countries where the hygiene standards are low and access to childcare, safe water and hygiene information are inadequate.

Moreover, this is of minimal importance in the western countries (for example, UK) where the infection rates are low, hygiene standards are better and breastfeeding mothers have access to information about childcare and hygiene. Furthermore, the functional healthcare system can be relied upon to treat any infection outbreaks in the country and prevent child deaths. Similarly, the only one piece of evidence relevant to babies in regard to 6 months of exclusive breastfeeding benefits in the United Kingdom is the slightly decreased risk of gastroenteritis, food allergies and iron deficiency anaemia which is linked to irreversible adverse mental, motor, and psychosocial outcomes [37]. This is important since the UK lacks a screening programme to detect such adverse population effects compared to the US which has a screening programme for iron deficiencies.

Most of the early complementary foods given to babies in developing countries are not nutrient dense to meet the energy requirements of the babies and do not provide adequate quantities of iron and zinc in the infant diets. Moreover, a study in eastern Kenya found that by age 3 months, $90 \%$ of infants were already receiving supplemental feedings of cow's milk and maize or millet gruels [38]. Cereals, roots and tubers account for more than three-quarters of energy intake and in some cases with small proportions of protein and micronutrient intakes [39]. Moreover, research evidence shows that infants and young children in sub-Saharan Africa subsist on gruel 


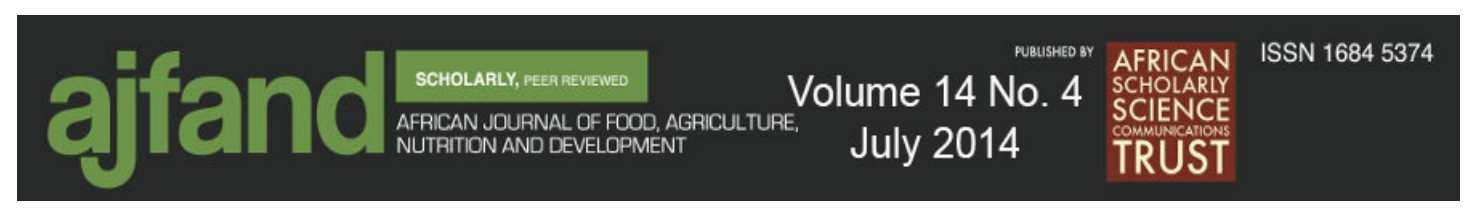

and porridge prepared from staples, accompanied with vegetables and legumes, and only occasionally, animal foods [40].

The low micronutrient densities in complementary diets, the early introduction of cow's milk, which is a potential source of mycotoxins, antibiotic residues, iron deficiency anaemia and the cow's milk ability to reduce the bioavailability of iron, zinc and vitamin $\mathrm{E}$ due to its high casein and calcium composition may thus be responsible for the high levels of malnutrition and micronutrient deficiencies in developing countries. Moreover, the small proportions of proteins in complementary foods and lack of animal source foods (red meat, fish and fowl) in most infant diets in developing countries may help explain why the 6 months of exclusive breastfeeding should not be reduced. Additionally, foods from plant sources have poor organoleptic qualities; contain anti-nutritional factors that further diminish appetites already suppressed by physiological nutrient deficiencies [41].

Breastfeeding promotion also needs to be considered. Women who receive skilled support and advice from health professionals on breastfeeding have a more positive breastfeeding experience and also breastfeed for longer [42]. Britton et al.[43] concluded that additional professional support has been found to help prolong exclusive breastfeeding . Furthermore, Dennis (2002) found that health professionals with a lack of knowledge of breastfeeding can be a negative source of support if they give inaccurate and inconsistent advice [44]. Women consistently report frustration with receiving conflicting advice from health professionals [45, 46]. A study by Leahy-Warren (2007) of first-time mothers found that two thirds relied on health professionals for information; however, nearly half of these mothers would have liked more information [47]. Any effort to increase rates of breastfeeding must take into account the knowledge and attitudes of health professionals and also their training needs. Thus, the need for sufficiently trained and competent health professionals is imperative to improve breastfeeding duration and increased rates of exclusive breastfeeding to 6 months.

Moreover, breastfeeding mothers in developing countries also lack key preparation skills and information needed to prepare health foods for the infants. However, the developed countries have a well developed food industry; have better access to health food preparation information for their infants as well as public health nutrition guidance. Moreover, micronutrient supplementation programmes for infants are more readily available in the developed countries unlike the developing countries. In addition, the food industry can be relied upon to produce safe and nutritious foods in the developed countries and the issue of 6 months of exclusive breastfeeding together with the health benefits (food safety issues) associated with it may not arise compared to the developing countries where the populations depend on food aid, consume foods that are low in micronutrient density and thereby reducing the 6 months of exclusive breastfeeding may have considerably more serious consequences. 


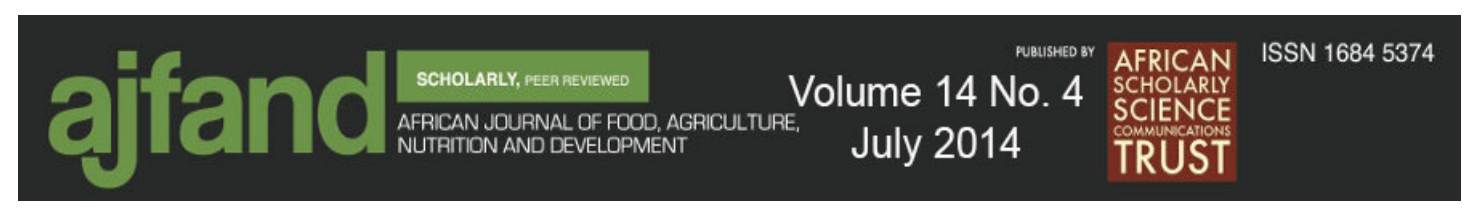

\section{CONCLUSION}

Breast milk remains the best and most secure form of nutrition for infants. The health benefits of exclusive breastfeeding to infants especially in developing countries should not be underrated. The introduction of the 6 months of exclusive breastfeeding recommendation policy was under-researched and had potential bias since it was based on observational studies. Questions about the effectiveness of breastfeeding policies and the conditions in the family, in the community, and in the society that enable women to breastfeed or prevent them from nursing their children are seriously under-researched and can only be addressed by not just looking at the benefits of breastfeeding to the infant but also at the benefits that exclusive breastfeeding extends to the mother.

Moreover, having a unified exclusive breastfeeding recommendation for both developing and developed countries remains an area for further research. The rate of infections (diarrhea, pneumonia, cough and flue) outbreaks, lack of access to information by breastfeeding mothers on infant hygiene and care, food preparation skills and the low micronutrient-density in complementary foods remain key infant feeding challenges in developing countries. To avert the problems of child malnutrition, morbidity and mortality, high rate of child infections that may arise from the lowering of the 6 months universal exclusive breastfeeding recommendation policy duration in developing countries, the 6 months of exclusive breastfeeding should instead be promoted in all developing countries and 4-6 months in all developed countries. The breastfeeding promotion programmes should be delivered by qualified and competent health professionals. The programmes should further highlight the importance of breastfeeding in relation to iron deficiency anaemia, child malnutrition, protection against infant infections, breastfeeding and food safety and hygiene and the health benefits that breastfeeding confers to the breastfeeding mothers.

Moreover, women's rights and status have been found to be of much importance for the prevention of childhood malnutrition. Now is the time to conduct research accordingly, on promoting the effects of breastfeeding that do not take into account the real duration of breastfeeding before setting up generalized new recommendations. This will reduce possible bias in the exclusive breastfeeding recommendation policy, confusion among breastfeeding mothers and reduce the incidences of malnutrition in developing countries. 


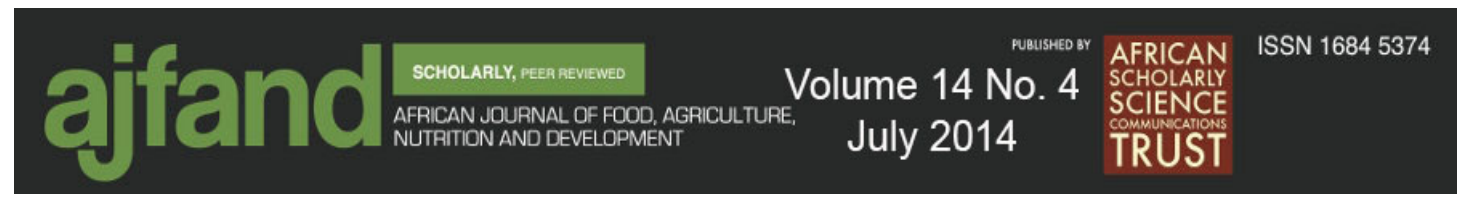

\section{REFERENCES}

1. World Health Organization. Global Strategy for Infant and Young Child Feeding: the Optimal Duration of Exclusive Breastfeeding. World Health Organization, Geneva. 2001.

2. Health Canada. Exclusive Breastfeeding Duration: Health Canada Recommendation. 2004. Available at: www.healthcanada.ca/nutrition.

3. Kramer MS, Chalmers B, Hodnett ED, Sevkovskaya Z, Dezikovich I and S Shapiro Promotion of breastfeeding intervention trial (PROBIT): a randomized trial in the Republic of Belarus. J. Am. Med. Assoc. 2001; 285 (4): 413-420.

4. Labbok MA Effects of breastfeeding on mother. Pediatr. Clinics. North America. 2001; 48: 143-158.

5. Kramer MS and $\mathbf{R}$ Kakuma The optimal duration of exclusive breastfeeding: a systematic review. World Health Organization (WHO/NHD/01.08; WHO/FCH/01.23). Geneva. 2001.

6. World Health Organisation. Global Data Bank on Infant and Young Child Feeding. 2009.

7. Butte N, Lopez-Alarcon MG and C Garza Nutrient adequacy of exclusive breastfeeding for the term infant during the first six months of life. WHO, Geneva. 2002; 153-178.

8. UNICEF. Progress for children: a world fit for children. Statistical Review Number 6. New York. 2007.

9. Alder EM, Williams FL, Anderson AS, Forsyth S, Du Florey CV and P Van der Velde What influences the timing of the introduction of solid food to infants? Brit. J. Nutr. 2004; 92: 527-531.

10. Hellin $\mathbf{K}$ and $\mathbf{G}$ Waller Mothers' mood and infant feeding: prediction of problems and practices. J. Reprod. Infant. Psychol. 1992; 10: 39-51.

11. Arora S, Mcjunkin C, Wehrer $\mathbf{J}$ and $\mathbf{P}$ Kuhn Major factors influencing breastfeeding rates: mother's perception of father's attitude and milk supply. Pediatr. 2000; 106 (5): 67-71.

12. De Olivieira MI, Camacho LA and AE Tedstone Extending breastfeeding duration through primary care: a systematic review of prenatal and postnatal interventions. J. Hum. Lact. 2001; 17: 326-343. 


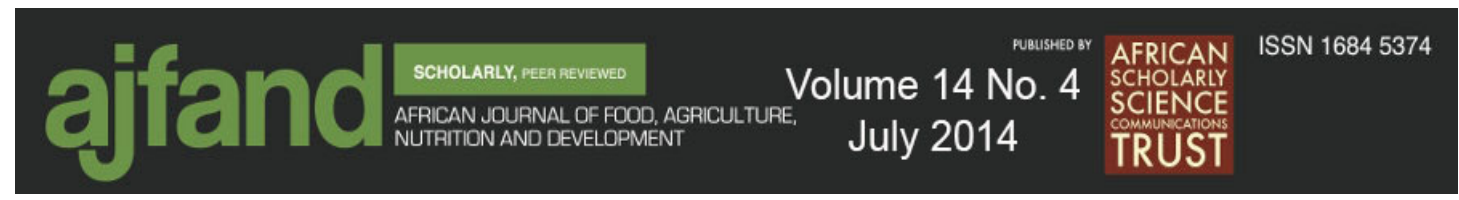

13. Taveras EM, Capra AM, Braveman PA, Jensvold NG, Escobar GJ and TA Lieu Clinician support and psychosocial risk factors associated with breastfeeding discontinuation. Pediatrics. 2003; 112 (1): 108-115.

14. World Health Organization. Nutrition: Information and attitudes among health personnel about early infant-feeding practices. WHO. Weekly. Epidem. 1995; 70: 117-120.

15. United Nations Children's Fund. Facts for Life. Wallingford. P and LA. 1993.

16. Lutter $\mathbf{C}$ Length of exclusive breastfeeding: linking biology and scientific evidence to a public health recommendation. J. Nutr. 2000; 130: 1335-1338.

17. Committee on Nutrition American Academy of Pediatrics Pediatric Nutrition Handbook. Am. Acad. Pediatr. Elk Grove Village, Illinois. 1998.

18. Sauls $\mathbf{H}$ Potential effect of demographic and other variables in studies comparing morbidity of breastfed and bottle-fed infants. Pediatr. 1979; 64: 523-527.

19. Hill A A Short Textbook of Medical Statistics. Hodder and Stoughton, London. 1977; 27.

20. Miettinen $\mathbf{O}$ The need for randomization in the study of intended effects. Stat. Med. 1983; 2: 267-271.

21. Bauchner $\mathbf{H}$, Leventhal $\mathbf{J}$ and $\mathbf{E}$ Shapiro Studies of breast-feeding and infections: how good is the evidence? JAMA. 1986; 256: 887-892.

22. Heinig M, Nommsen L, Peerson J, Lonnerdal B and K Dewey Intake and growth of breastfed and formula-fed infants in relation to the timing of introduction of complementary foods: the darling study. Acta. Paediatr. Scand. 1993; 82: 999-1006.

23. Cohen R, Brown K, Canahuati J, Rivera $\mathbf{L}$ and $\mathbf{K}$ Dewey Effects of age of introduction of complementary foods on infant breast milk intake, total energy intake, and growth: a randomized intervention study in Honduras. Lanc. 1994; 344: 288-293.

24. Dewey $\mathbf{K}$, Cohen $\mathbf{R}$, Brown $\mathbf{K}$ and $\mathbf{L}$ Rivera Age of introduction of complementary foods and growth of term, low-birth-weight, breast-fed infants: a randomized intervention study in Honduras. Am. J. Clin. Nutr. 1999; 69: 679-686. 


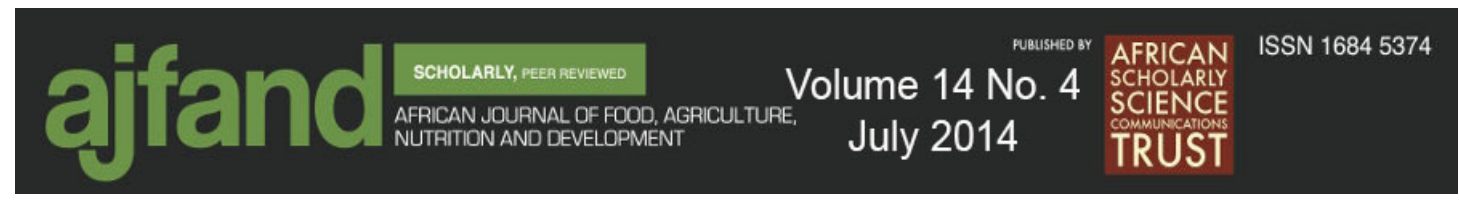

25. Frongillo EJr and JP Habicht Investigating the weanling's dilemma: lessons from Honduras. Nutr. Rev. 1997; 55: 390-395.

26. Horta BL, Bahl R, Martinés JC and CG Victoria Evidence on the longterm effects of breastfeeding: systematic reviews and meta-analysis. WHO, Geneva. 2007. Available at: http://whqlibdoc.who.int/publications/2007/9789241595230_eng.pdf. Accessed June 2012.

27. Kawai T, Goto A, Watanabe E, Nagasawa $\mathbf{M}$ and $\mathbf{S}$ Yasumura Lower respiratory tract infections and gastrointestinal infections among mature babies in Japan. Pediatr. Int. 2010.

28. De Onis M, Garza C, Victora CG, Bhan MK and KR Norum The WHO Multicentre Growth Reference Study (MGRS): rationale, planning, and implementation. Food. Nutr. Bull. 2004; 25 (1): 3-84.

29. Agostoni C, Braegger C, Decsi T, Kolacek S, Koletzko B and KF Michaelsen Breast-feeding: a commentary by the ESPGHAN Committee on Nutrition. J. Pediatr. Gastroenterol. Nutr. 2009; 49: 112-25.

30. Strassburger SZ, Vitolo MR, Bortolini GA, Pitrez PM, Jones MH and RT Stein Nutritional errors in the first months of life and their association with asthma and atopy in preschool children. J. Pediatr. 2010; 86 (5): 391-9.

31. Silvers KM, Frampton CM, Wickens K, Epton MJ, Pattemore PK and T Ingham New Zealand Asthma and Allergy Cohort Study Group: breastfeeding protects against adverse respiratory outcomes at 15 months of age. Matern. Child. Nutr. 2009; 5 (3): 243-50.

32. Duijts L, Jaddoe VW, Hofman A and $\mathbf{H}$ Moll Prolonged and exclusive breastfeeding reduces the risk of infectious diseases in infancy. Pediatrics. 2010; 126 (1): 18-25.

33. Midodzi WK, Rowe BH, Majaesic CM, Saunders LD and A Senthilselvan Early life factors associated with incidence of physician-diagnosed asthma in preschool children: results from the Canadian Early Childhood Development cohort study. J. Asthma. 2010; 47 (1): 7-13.

34. Walker $\mathbf{M}$ International breastfeeding initiatives and their relevance to the current state of breastfeeding in the United States. J. Midwif. Womens. Health. 2007; 52 (6): 549-55.

35. Phillips G, Brett $\mathbf{K}$ and $\mathbf{P}$ Mendola Previous breastfeeding practices and duration of exclusive breastfeeding in the United States. Matern. Child. Health. J. 2010. 


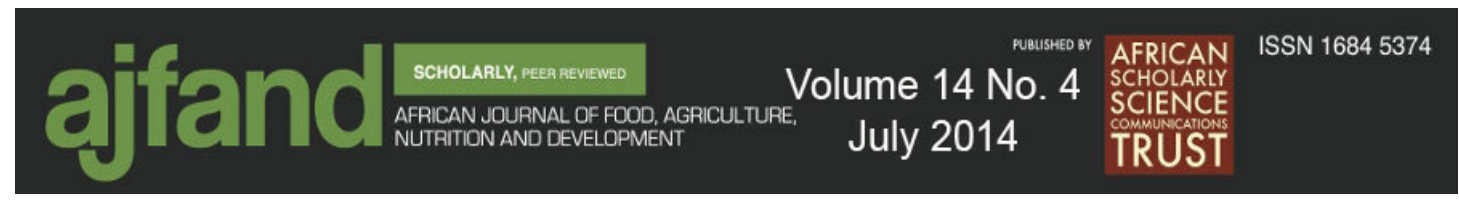

36. Spiby H, McCormick F, Wallace L, Renfrew MJ, D’Souza L and L Dyson A systematic review of education and evidence-based practice interventions with health professionals and breastfeeding counsellors on duration of breast feeding. Midwif. 2009; 25 (1): 50-61.

37. Fewtrell M, Wilson DC, Booth I and A Lucas When to wean: how good is the evidence for six months' exclusive breastfeeding. BMJ. 2011; 342: 234.

38. Allen LH The Nutrition CRSP: what is marginal malnutrition and does it affect human function? Nutr. Rev. 1993; 51: 255-267.

39. Tarini A, Seidou $\mathbf{B}$ and $\mathbf{H}$ Delisle La qualite' nutritionelle globale de l'alimentation d'enfants nige'riens se refle`te sur leur croissance. Cahiers Sante'. 1999; 9: 23-31.

40. Faber M, Smuts CM and AJS Benade' Dietary intake of primary school children in relation to food production in a rural area in KwaZulu-Natal, South Africa. Int. J. Food. Sci. Nutr. 1999; 50: 57-64.

41. Golden MHN The nature of nutritional deficiency in relation to growth failure and poverty. Acta. Paediatr. Scand. 1991; 374: 95-110.

42. Sikorski J, Renfrew MJ, Pindoria S and A Wade Support for breastfeeding mothers: a systematic review. Paed. Perinatal. Epidem. 2003; 17: 407-417

43. Britton C, McCormick FM, Renfrew MJ, Wade A and SE King Support for breastfeeding mothers. Cochrane Database of Systematic Reviews; Issue 1. Art. No.: CD001141. 2007.

44. Dennis CL Breastfeeding initiation and duration: a 1990-2000 literature review. J. Obst. Gynec. Neonatal. Nursing. 2002; 31: 12-32.

45. Simmons V Exploring inconsistent breastfeeding advice: 2. Brit. J. Midwif. 2002; 10: 616-619

46. Smale M, Renfrew MJ, Marshall JL and $\mathbf{H}$ Spiby Turning policy into practice: more difficult than it seems. The case of breastfeeding education. Matern. Child. Nutr. 2006; 2: 103-113.

47. Leahy-Warren P Social support for first-time mothers: an Irish study. MCN: Am. J. Matern. Child. Nursing. 2007; 36: 368-374. 